\title{
TEMPORAL BEHAVIOR OF SOLAR P-MODES FROM GONG AND GOLF EXPERIMENTS
}

\author{
V.G. GAVRYUSEV \\ CAISMI CNR, Largo E.Fermi 5, 50125, Florence, Italy
}

\begin{abstract}
AND
E.A. GAVRYUSEVA

Osservatorio Astronomico di Capodimonte, via Moiariello 16, I-80131, Naples, Italy

Institute for Nuclear Research of RAN, Moscow, Russia
\end{abstract}

We used the measurements of solar oscillations taken by GONG and GOLF experiments. The first set of data are the integrated images obtained from the complex GONG observations taken from June 10 of 1995 to January 7 of 1997, 578 days in total, referenced below as ts 0 time series. Radial, dipole and quadrupole modes are well visible in this time series. The second data set is the GOLF time series obtained onboard SOHO mission from April 11, 1996 to June 22, 1997. GOLF observes the "Sun as a star". This time series is similar to ts0 of GONG but of a better quality (better signal-to-noise ratio; uniform, practically uninterrupted data). Both experiments are significantly overlapped in time. Because of this the direct comparison between them is possible, and the effects visible in both observations support each other.

To study the temporal evolution of the power of a given mode of the oscillations, one has to analyze the sequence of the corresponding power spectra. A 20 day window, which is reasonably close to the typical visibility time of all radial modes of radial order from 13 to 26 (Gavryusev \& Gavryuseva, 1996), was chosen. This window runs through the whole data series with a one day step. The standard Fourier transform produces the mean power corresponding to the current window.

The temporal change of the power of each individual mode usually shows a quasi random appearance of the pulses of different duration and amplitude (Baudin 1996; Gavryusev \& Gavryuseva 1997). Such behavior is typical for all the modes we have analyzed. Components of the rotational multiplets of a given radial order do not perfectly correlate. Most of them differ significantly in their mean power level over 2.5 years. As a result, the components of the rotational multiplets are strongly asymmetric.

There is a question still to be answered, whether the big pulses appear completely randomly in time, or if there is something coordinating their appearance in a more or less regular manner. One of the current theoretical hypotheses is the following: the big pulses appear just by chance, due to random interference between very frequent excitation events. In such a case one can not expect any stable periodicity in the time 
sequence of the spectra of the window running throw the long data set. It seems true for some of the modes. But we find a few examples of very regular periodical behavior over 800 days. $\ell=0 n=15$ and $n=17$ demonstrate quasi 50-days and 135-days variations respectively. The other modes behave in a different way. But for many of them the autocorrelation analysis shows the presence of significant periodicities.

To reveal something common for all the modes, we calculated the sum of the power of 14 modes of radial order from 13 to 26 . The frequencies of the individual peaks in the spectra of each running window generally change with time, and in many cases there is more than one peak around the frequency of an expected mode. Because of this we used the power integrated in $7 \mu \mathrm{Hz}$ intervals, centered at the mode frequency, obtained from the mode profile revealed from the complete data series.

A variation of the total $p$-mode power with the period of solar rotation was clearly detected by Gavryusev \& Gavryuseva $(1996,1997)$ in IPHIR measurements (taken in 1988 , when solar activity was rising up). During the time of solar minimum, covered by the GONG and GOLF data sets, this periodicity is much less visible in the running spectra, while it can still be detected by a Fourier transform of the series of the power spectra or by their autocorrelation analysis in the both experiments. It seems, that during this time the solar magnetic structure has changed and the new structure is not yet established. We may expect, that later on, when the new magnetic structure will be more regular, the change of $p$-mode power will become stronger again and better correlated with solar rotation.

It is necessary to investigate this question in detail, but one thing is already obvious: the simple model of completely random frequent excitation fails to explain such long term correlations in time. The excitation processes are stochastic but by no means trivial. And the investigation of the temporal behavior of the $p$-mode power is of vital necessity if we wish to understand the mechanisms of the excitation of the solar oscillations.

\section{Acknowledgements}

This work utilizes data obtained by the Global Oscillation Network Group (GONG) project, managed by the National Solar Observatory, a Division of the National Optical Astronomy Observatories, which is operated by AURA, Inc. under a cooperative agreement with the National Science Foundation.

The data were acquired by instruments operated by the Big Bear Solar Observatory, High Altitude Observatory, Learmonth Solar Observatory, Udaipur Solar Observatory, Instituto de Astrofísico de Canarias, and Cerro Tololo Interamerican Observatory.

\section{References}

Baudin F., Gabriel A., Gilbert D., Palle P., Régulo C., 1996, A\&A, 311, 1024

Gavryusev V.G., Gavryuseva E.A., 1996, A\&A, 310, 651

Gavryusev V.G., Gavryuseva E.A., 1996, in Cool Stars, Stellar Systems, and the Sun 95, ASP Conference Series, v. 109, 129

Gavryusev V.G., Gavryuseva E.A., 1997, Solar Physics, 172, 27 